\title{
An Upper Bound of the Throughput for Multi-Radio Wireless Mesh Networks
}

\author{
Rong-Hong Jan, Shu-Ying Huang, and Chu-Fu Wang
}

\begin{abstract}
This paper focuses on how to determine an upper bound of maximum throughput from mesh clients to the Internet (or from the Internet to mesh clients) for multi-radio Wireless Mesh Networks (WMNs) under an interference-free assumption. In the case of the number of channels for assignment being high enough in a multi-radio WMN to meet the interference-free assumption, then the resulting solution can provide the basis for channel assignment and routing to achieve optimal throughput.
\end{abstract}

Index Terms-Multi-radio wireless mesh networks, maximum flow problem, throughput.

\section{INTRODUCTION}

$\mathbf{M}$ ULTI-RADIO WMNs are the most promising networking technology recently to extend last-mile broadband Internet access. Due to the fact that a multi-radio WMN contains no wired infrastructure within its serving field, it has a low cost of deployment and maintenance. It is therefore attractive to several wireless network applications, e.g., wireless last mile access of ISPs, broadband home networking, community and neighborhood networks, enterprise networking, building automation, and so on [1]. A multi-radio WMN consists of mesh routers and mesh clients where mesh routers have minimal mobility and form the wireless backbone through wireless links. Other than their routing functionality, mesh routers provide additional functions to support mesh networking. With access point functionality, mesh routers can provide network access for mesh clients within their coverage area. With gateway functionality, mesh routers can connect to the wired Internet. Mesh routers can thus be classified into three types, i.e., pure routers, mesh gateways, and mesh APs (see Fig. 1(a)). Each mesh router is equipped with multiple radio interfaces for effective use of available orthogonal channels. Thus, they can reduce wireless interference and increase network throughput [2]. Two of the most challenging research issues in multi-radio WMNs are the channel assignment and the routing problems, which are usually coupled together to maximize network throughput. The channel assignment problem determines the connectivity between nodes, thus the network topology of the WMN is then determined. Based on the resulting network topology, routing decisions can also

Manuscript received January 6, 2010. The associate editor coordinating the review of this letter and approving it for publication was H.-H. Chen.

R.-H. Jan and S.-Y. Huang are with the Department of Computer Science, National Chiao Tung University, Hsinchu, 300, Taiwan, ROC.

C.-F. Wang (corresponding author) is with the Department of Computer Science, National Pingtung University of Education, Pingtung, 900, Taiwan, ROC (e-mail: cfwang@mail.npue.edu.tw).

This paper was supported in part by the National Science Council of the ROC, under grant NSC 97-2221-E-009-049-MY3. The authors would also like to thank Dr. An-Kai Jeng for his helpful assistance in conducting the simulation results.

Digital Object Identifier 10.1109/LCOMM.2010.08.100023

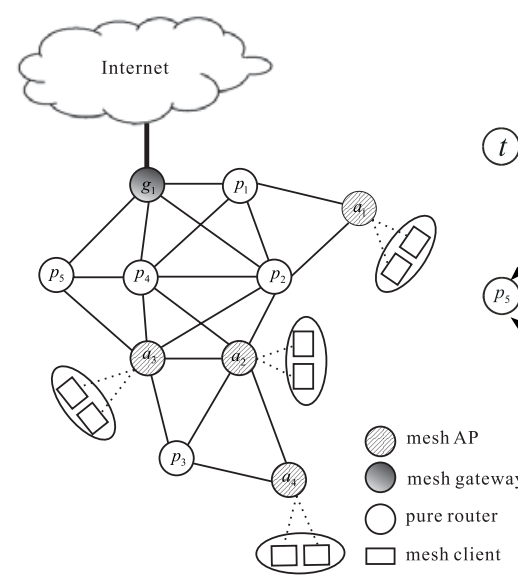

(a)

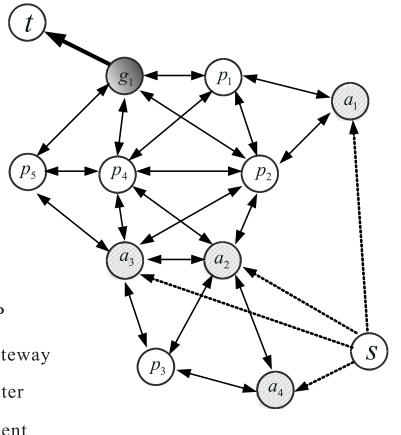

(b)
Fig. 1. An example for illustrating the problem formulation in multi-radio WMNs.

be made. However, in order to achieve better results, these two optimization problems should be considered jointly, not sequentially [3]. Unfortunately, the joint problem of finding optimal throughput is NP-hard [4].

In this paper, we want to find an upper bound of the throughput for multi-radio WMNs. More precisely, our problem is that, given the deployment of mesh routers and the number of radio interfaces of each mesh router, we want to find the maximum flow from mesh clients to the wired Internet or from the wired Internet to the mesh clients under an interferencefree assumption. Since these two cases are symmetric, we only consider the previous case in this paper.

\section{Problem Definition}

The sets of the pure routers, the mesh gateways and the mesh APs are denoted as $V_{P}, V_{G}$, and $V_{A}$, respectively. The network model for finding the maximum throughput from mesh clients to the wired Internet in the multi-radio WMN under an interference-free assumption can be modeled as a directed graph $G=(V, E)$, called the communication graph, where $V$ is a set of nodes containing all mesh routers plus a source $s$ and a sink $t$; that is $V=V_{P} \cup V_{G} \cup V_{A} \cup\{s, t\}$. The source $s$ represents all mesh clients, and the sink $t$ represents the wired Internet. Given any two mesh routers, if the distance between them is less than the transmission radius (we assume that all interfaces have identical transmission radii), there are two directed edges with opposite directions between them. We add an edge from $s$ to every mesh AP and also add an edge from every mesh gateway to $t$ (the corresponding communication graph of Fig. 1(a) is shown in Fig. 1(b)). Now, we will 
formulate the maximum throughput problem of a multi-radio WMN as a network flow-like problem, called the Augmenting Network Flow Problem (ANFP) in communication graph $G$.

Let $R_{i}^{B}$ be the number of backhaul interfaces equipped in mesh router $i$ for backbone communication, $R_{i}^{C}$ be the number of client interfaces of mesh AP $i, c_{w}$ be the capacity of the wired link and $C$ be the channel capacity. Thus, the maximum capacity of mesh router (mesh AP) $i$ is equal to $R_{i}^{B} \times C$ $\left(R_{i}^{C} \times C\right)$, respectively. The edge capacity $c_{i j}$ for each edge $(i, j) \in E$ can then be set as follows:

$c_{i j}= \begin{cases}\min \left\{R_{i}^{B}, R_{j}^{B}\right\} \times C, & \text { if } i, j \in V-\{s, t\} \\ R_{j}^{C} \times C, & \text { if } i=s, \text { and } j \in V-\{s, t\} \\ c_{w}, & \text { if } i \in V-\{s, t\} \text { and } j=t,\end{cases}$

The flow on $G$ must satisfy two types of basic constraints, i.e., the edge capacity constraint and the node capacity constraint. Let $x_{i j}$ denote the flow on edge $(i, j)$. The edge capacity constraint ensures that the flow on each link cannot exceeded the capacity of the edge and the property of flow conservation. For the node capacity constraint on each mesh router, the sum of incoming flows and the sum of outgoing flows must not exceed its capacity, i.e., its backhaul interfaces multiplied by channel capacity. Therefore, our problem ANFP can be mathematically formulated as follows:

Maximize $f$

Subject to

$$
\begin{gathered}
\sum_{j \in V} x_{i j}-\sum_{k \in V} x_{k i}= \begin{cases}f, & \text { if } i=s \\
0, & \text { if } i \in V-\{s, t\}, \forall i \in V \\
-f, & \text { if } i=t\end{cases} \\
0 \leq x_{i j} \leq c_{i j}, \forall i, j \in V \\
\sum_{k \in V} x_{k i}+\sum_{j \in V} x_{i j} \leq R_{i}^{B} \times C, \forall i \in V_{P} \\
\sum_{k \in V} x_{k i}+\sum_{j \in V-\{t\}} x_{i j} \leq R_{i}^{B} \times C, \forall i \in V_{G} \\
\sum_{k \in V-\{s\}} x_{k i}+\sum_{j \in V} x_{i j} \leq R_{i}^{B} \times C, \forall i \in V_{A}
\end{gathered}
$$

\section{iII. Problem Transformation}

In this section, we present how to transform the problem ANFP into a maximum flow problem. Comparing problem ANFP to the maximum flow problem, problem ANFP has additional node capacity constraints (4)-(6). Our approach is to transform each of the constraints (4)-(6) into a set of flow conservation constraints and edge capacity constraints using node splitting on sets $V_{P}, V_{G}$, and $V_{A}$, respectively. As shown in Fig. 2(a), we split each pure router node $i \in V_{P}$ into two connecting nodes $i_{\text {in }}$ and $i_{\text {out }}$. Node $i_{\text {in }}$ has an edge entering it for every edge entering $i$, while node $i_{\text {out }}$ has an edge leaving it for every edge leaving $i$. We call the resulting graph $G_{P}^{\prime}=\left(V_{P}^{\prime}, E_{P}^{\prime}\right)$ an augmenting communication graph with pure router nodes splitting. The constraint (4) in ANFP can be rewritten as follows:

$$
\begin{aligned}
& \sum_{k \in V} x_{k i}+\sum_{j \in V} x_{i j} \leq R_{i}^{B} \times C, \forall i \in V_{P} \\
\Rightarrow \quad & \sum_{k \in V} x_{k i}+\sum_{k \in V} x_{k i} \leq R_{i}^{B} \times C, \forall i \in V_{P} \\
& \text { (by constraint }(2)) \\
\Rightarrow \quad & \sum_{k \in V} x_{k i} \leq\left(R_{i}^{B} \times C\right) / 2, \forall i \in V_{P}
\end{aligned}
$$
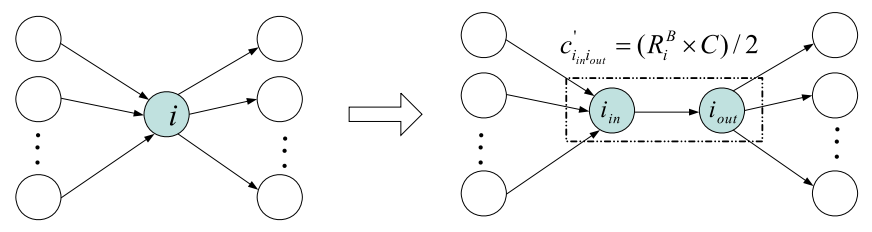

(a)
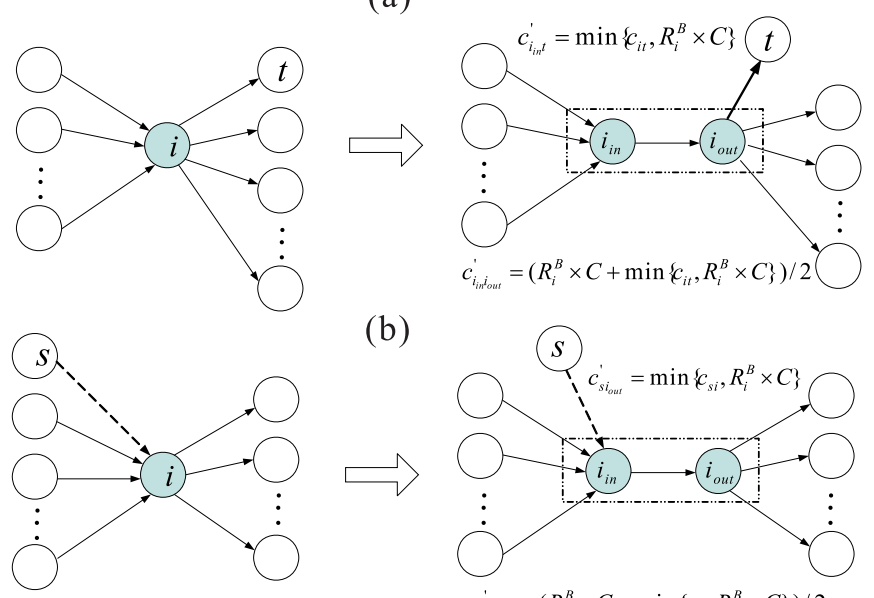

(b)

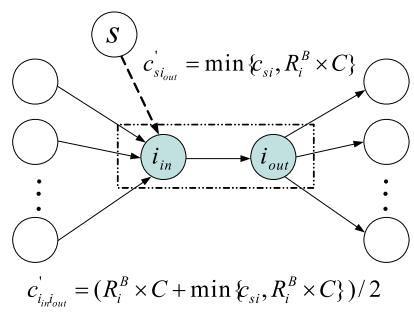

(c)

Fig. 2. The illustration of nodes splitting.

Let flow $x_{i_{\text {in }} i_{\text {out }}}^{\prime}$ on edge $\left(i_{\text {in }}, i_{\text {out }}\right) \in E_{P}^{\prime}$ be $\sum_{k \in V} x_{k i}$. Inequation (7) can be rewritten as $x_{i_{\text {in }} i_{\text {out }}}^{\prime} \leq\left(R_{i}^{B} \times C\right) / 2, \forall i \in$ $V_{P}$ (an edge capacity constraint). The flow $x_{i_{\text {in }} i_{\text {out }}}^{\prime}$ can be rewritten as $x_{i_{\text {in }} i_{\text {out }}}^{\prime}-\sum_{k \in V} x_{k i}=0$ (a flow conservation constraint). And by constraint (2), the flow $x_{i_{\text {in }} i_{\text {out }}}^{\prime}$ can also be rewritten as $\sum_{j \in V} x_{i j}-x_{i_{\text {in }} i_{\text {out }}}^{\prime}=0$ (the flow conservation constraint). Thus, constraint (4) can be replaced by two flow conservation constraints and an edge capacity constraint. If we set capacity of edge $\left(i_{\text {in }}, i_{\text {out }}\right), \forall i \in V_{P}$ in $G_{P}^{\prime}$ to $\left(R_{i}^{B} \times C\right) / 2$ and set the other edges' in $G_{P}^{\prime}$ to $c_{i j}$, then, we have the following lemma.

Lemma 1. A flow meets the constraint (2)-(4) in $G$ of the problem ANFP, which also meets the flow conservation constraints and the edge capacity constraints in $G_{P}^{\prime}$.

Now, we define the augmenting communication graph $G_{G}^{\prime}=$ $\left(V_{G}^{\prime}, E_{G}^{\prime}\right)$ with gateway router nodes splitting as follows. Each mesh gateway node $i$ in $G$ is split into two connecting nodes $i_{\text {in }}$ and $i_{\text {out }}$. The node $i_{\text {in }}$ has an edge entering it for every edge entering $i$. The node $i_{\text {out }}$ has an edge leaving it for every edge leaving $i$ (see Fig. 2(b)). The constraint (5) in ANFP can be rewritten as follows:

$$
\begin{aligned}
& \sum_{k \in V} x_{k i}+\sum_{j \in V-\{t\}} x_{i j} \leq R_{i}^{B} \times C, \forall i \in V_{G} \\
\Rightarrow & \sum_{j \in V} x_{i j}+\left(\sum_{j \in V} x_{i j}-x_{i t}\right) \leq R_{i}^{B} \times C, \forall i \in V_{G} \\
\Rightarrow \quad & \sum_{j \in V} x_{i j} \leq\left(R_{i}^{B} \times C+x_{i t}\right) / 2, \forall i \in V_{G}
\end{aligned}
$$

Note that $x_{i t} \leq \min \left\{c_{i t}, R_{i}^{B} \times C\right\}$. Then, inequation (8) can be rewritten as $\sum_{j \in V} x_{i j} \leq\left(R_{i}^{B} \times C+\min \left\{c_{i t}, R_{i}^{B} \times\right.\right.$ $C\}) / 2, \forall i \in V_{G}$. Similarly, let flows $x_{i_{\text {in }} i_{\text {out }}}^{\prime}=\sum_{k \in V} x_{k i}$ and $x_{i_{\text {out }} t}^{\prime}=x_{i t}$. We replace constraint (5) by a capacity constraint $x_{i_{\text {in }} i_{\text {out }}}^{\prime} \leq\left(R_{i}^{B} \times C+\min \left\{c_{i t}, R_{i}^{B} \times C\right\}\right) / 2, \forall i \in V_{G}$ and two flow conservation constraints $\sum_{k \in V} x_{k i}-x_{i_{\text {in }} i_{\text {out }}}^{\prime}=0$ and $x_{i_{\text {in }} i_{\text {out }}}^{\prime}-\left(x_{i_{\text {out }} t}^{\prime}+\sum_{j \in V-\{t\}} x_{i j}\right)=\sum_{k \in V} x_{k i}-\left(x_{i t}+\right.$ 


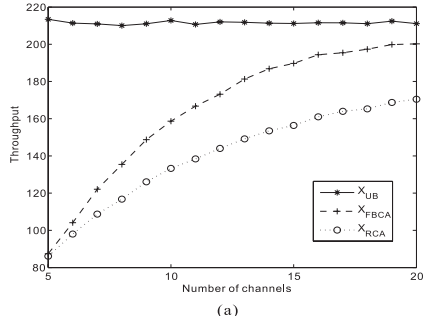

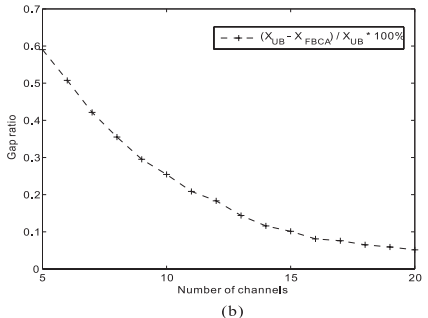

Fig. 3. Numerical results.

$\left.\sum_{j \in V-\{t\}} x_{i j}\right)=\sum_{k \in V} x_{k i}-\sum_{j \in V} x_{i j}=0$. If we set the capacity of the edge $\left(i_{\text {in }}, i_{\text {out }}\right), \forall i \in V_{G}$ in $G_{G}^{\prime}$ to $\left(R_{i}^{B} \times C+\min \left\{c_{i t}, R_{i}^{B} \times C\right\}\right) / 2$, and set the capacity of the edge $\left(i_{\text {out }}, t\right)$ to $\min \left\{c_{i t}, R_{i}^{B} \times C\right\}$, and set the other edges in $G_{G}^{\prime}$ to $c_{i j}$, then, we have the following lemma.

Lemma 2. A flow meets constraint (2)-(3) and (5) in $G$ of the problem ANFP, which also meets the flow conservation constraints and the edge capacity constraint in $G_{G}^{\prime}$.

Similarly, constraint (6) can be transformed by two flow conservation constraints and a capacity constraint in the augmenting communication graph $G_{A}^{\prime}=\left(V_{A}^{\prime}, E_{A}^{\prime}\right)$ with mesh AP router nodes splitting (see Fig. 2(c)). If we set the capacity of the edge $\left(i_{\text {in }}, i_{\text {out }}\right), \forall i \in V_{A}$ in $G_{A}^{\prime}$ to $\left(R_{i}^{B} \times C+\min \left\{c_{s i}, R_{i}^{B} \times C\right\}\right) / 2$, and set the capacity of the edge $\left(s, i_{i n}\right)$ to $\min \left\{c_{s i}, R_{i}^{B} \times C\right\}$, and set the other edges in $G_{A}^{\prime}$ to $c_{i j}$, we have the following lemma.

Lemma 3. A flow meets constraint (2)-(3) and (6) in $G$ of the problem ANFP, which also meets the flow conservation constraints and the edge capacity constraint in $G_{A}^{\prime}$.

Let $G_{P G A}^{\prime}=\left(V_{P G A}^{\prime}, E_{P G A}^{\prime}\right)$ be the resulting augmenting communication graph with vertex sets $V_{P}, V_{G}$, and $V_{A}$ splitting. And let the capacity $c_{i j}^{\prime}$ of edge $(i, j)$ in $G_{P G A}^{\prime}$ be the values defined in Lemmas 1-3 (see Fig. 2). Then we have a network flow problem on graph $G_{P G A}^{\prime}$. By Lemmas 1-3, we have the following theorem.

Theorem 4. The problem ANFP in graph $G$ can be transformed into the network flow problem in $G_{P G A}^{\prime}$.

\section{AnAlysis}

Now, we give the time complexity analysis for solving the network flow problem in $G_{P G A}^{\prime}$. Note that the cost of splitting every mesh router $i$ into $i_{\text {in }}$ and $i_{\text {out }}$, to obtain $V_{P G A}^{\prime}$ is $O(|V|)$. The cost of adding a new edge $\left(i_{\text {in }}, i_{\text {out }}\right)$ and assigning its capacity, repeated $|V|$ times, is also $O(|V|)$. The total cost of this construction is therefore $O(|V|)$. Note that $\left.\left|V_{P G A}^{\prime}\right|\right)=2|V|$ and $\left|E_{P G A}^{\prime}\right|=|E|+|V|$, if we apply the Edmonds-Karp algorithm to the graph $G_{P G A}^{\prime}$. Hence, the total cost of finding a maximum flow in the original graph $G=(V, E)$ is $O(|V|)+O\left(\left|V_{P G A}^{\prime}\right|\left|E_{P G A}^{\prime}\right|^{2}\right)=O(2|V|(|E|+$ $\left.|V|)^{2}\right)$.

On the other hand, we have conducted simulations to demonstrate how tight the proposed throughput upper bound is. In the simulations, we observe the gap between the throughput upper bound $\left(X_{U B}\right)$ and the solution values (the lower bounds) found by two heuristic channel assignment algorithms, the flow-based channel assignment (FBCA) algorithm and the random channel assignment (RCA) algorithm, respectively. The FBCA algorithm uses a greedy approach to assign channels. At first, it ignores the channel interference constraint and performs the maximum-flow algorithm on the communication graph $G$ to determine the possible total incoming load of each node. Then, it assigns the remaining available channels to the interfaces of nodes one by one in non-increasing order of the load value of each node. For the RCA algorithm, the channels are randomly assigned to each node's interfaces. The solution values found by the FBCA algorithm and the RCA algorithm are denoted by $X_{F B C A}$ and $X_{R C A}$, respectively. In the simulations, we consider an $8 \times 8$ grid network. For each topology, we choose 30 mesh APs and 6 mesh gateways randomly. The remaining nodes are pure routers. Each pure router (mesh gateway) is equipped with 2 (5, respectively) backhaul interfaces. Each mesh AP is equipped with 2 backhaul interfaces and 1 client interface. The capacity of each channel is $10 \mathrm{Mbps}$ and the capacity of the wired link is $100 \mathrm{Mbps}$. Fig. 3(a) shows the effects of the number of channels on the network throughput. Each data point in Fig. 3(a) is the average over the 1000 topologies. Note that $X_{F B C A}$ and $X_{R C A}$ can serve as the lower bounds of the optimal throughput and $X_{F B C A}$ is better than $X_{R C A}$. Thus, the optimal throughput is guaranteed to fall between $X_{U B}$ and $X_{F B C A}$. Fig. 3(b) shows the gap ratios of the value $X_{F B C A}$ to the upper bound $X_{U B}$ where the gap ratio is defined to be $\left(X_{U B}-X_{F B C A}\right) / X_{U B} \times 100 \%$. From Fig. 3 (b), we learn that the gap ratio is less than $10 \%$ if the number of available channels is greater than 15 . This means that our proposed throughput upper bound $X_{U B}$ is close to the optimal throughput if the number of available channels is greater than 15.

\section{CONClusion}

In this paper, given the deployment of mesh routers and the number of radio interfaces of each mesh router, we want to find the maximum flow from mesh clients to the wired Internet under an interference-free assumption. We define the maximum throughput of the problem as an upper bound of the throughput for the given wireless mesh network. The proposed problem is transformed into a maximum flow problem, and then the problem can be solved by existing maximum flow algorithms. Therefore, an upper bound of the throughput for the given wireless mesh network can be obtained in polynomial time.

\section{REFERENCES}

[1] I. F. Akyildiz, X. D. Wang, and W. L. Wang, "Wireless mesh networks: a survey," Computer Networks, vol. 47, pp. 445-487, 2005.

[2] A. Raniwala, K. Gopalan, and T. C. Chiueh, "Centralized channel assignment and routing algorithms for multi-channel wireless mesh networks," ACM SIGMOBILE Mobile Computing and Commun. Rev., vol. 8, pp. 50-65, 2004.

[3] J. Chen, S. He, Y. Sun, P. Thulasiramanz, and X. Shen, "Optimal flow control for utility-lifetime tradeoff in wireless sensor networks," Computer Networks, vol. 53, pp. 3031-3041, 2009.

[4] K. Jain, J. Padhye, V. N. Padmanabhan, and L. Qiu, "Impact of interference on multi-hop wireless network performance," in Proc. 9th Annual International Conference on Mobile Computing and Networking, pp. 66-80, 2003. 